\title{
Anti-Osteoporosis Medication Prescriptions and Incidence of Subsequent Fracture Among Primary Hip Fracture Patients in England and Wales: An Interrupted Time-Series Analysis
}

\author{
Samuel Hawley, ${ }^{1}$ Jose Leal, ${ }^{2}$ Antonella Delmestri, ${ }^{1}$ Daniel Prieto-Alhambra, ${ }^{1,3,4,5}$ Nigel K Arden, ${ }^{1,3}$ \\ Cyrus Cooper, ${ }^{1,3}$ M Kassim Javaid, ${ }^{1,3 *}$ and Andrew Judge ${ }^{1,3 *}$; for the REFReSH Study Group \\ ${ }^{1}$ Oxford National Institute for Health Research (NIHR) Musculoskeletal Biomedical Research Unit, Nuffield Department of Orthopaedics, \\ Rheumatology and Musculoskeletal Sciences, University of Oxford, Oxford, UK \\ ${ }^{2}$ Health Economics Research Centre, Nuffield Department of Population Health, University of Oxford, Oxford, UK \\ ${ }^{3}$ Medical Research Council (MRC) Lifecourse Epidemiology Unit, University of Southampton, Southampton General Hospital, Southampton, UK \\ ${ }^{4}$ Hospital del Mar Medical Research Institute (IMIM), Universitat Autònoma de Barcelona and Red Tematica de Investigacion Cooperativa en \\ Envejecimiento y Fragilidad (RETICEF), Instituto de Salud Carlos III, Barcelona, Spain \\ ${ }^{5}$ Grup de Recerca en Malalties Prevalents de I'Aparell Locomotor (GREMPAL) Research Group, Institut d'Investigacio en Atencio Primaria (IDIAP) \\ Jordi Gol Primary Care Research Institute, Universitat Autònoma de Barcelona, Barcelona, Spain
}

\begin{abstract}
In January 2005, the National Institute for Health and Care Excellence (NICE) in England and Wales provided new guidance on the use of antiosteoporosis therapies for the secondary prevention of osteoporotic fractures. This was shortly followed in the same year by market authorization of a generic form of alendronic acid within the UK. We here set out to estimate the actual practice impact of these events among hip fracture patients in terms of antiosteoporosis medication prescribing and subsequent fracture incidence using primary care data (Clinical Practice Research Datalink) from 1999 to 2013. Changes in level and trend of prescribing and subsequent fracture following publication of NICE guidance and availability of generic alendronic acid were estimated using an interrupted time series analysis. Both events were considered in combination within a 1-year "intervention period." We identified 10,873 primary hip fracture patients between April 1999 and Sept 2012. Taking into account prior trend, the intervention period was associated with an immediate absolute increase of $14.9 \%(95 \% \mathrm{Cl}, 10.9$ to 18.9$)$ for incident antiosteoporosis prescriptions and a significant and clinically important reduction in subsequent major and subsequent hip fracture: $-0.19 \%(95 \% \mathrm{Cl},-0.28$ to -0.09$)$ and $-0.17 \%(95 \% \mathrm{Cl},-0.26$ to -0.09$)$ per 6 months, respectively. This equated to an approximate $14 \%$ (major) and $22 \%$ (hip) reduction at 3 years postintervention relative to expected values based solely on preintervention level and trend. We conclude that among hip fracture patients, publication of NICE guidance and availability of generic alendronic acid was temporally associated with increased prescribing and a significant decline in subsequent fractures. (c) 2016 American Society for Bone and Mineral Research.
\end{abstract}

KEY WORDS: EPIDEMIOLOGY; OSTEOPOROSIS; HIP FRACTURE; SECONDARY PREVENTION; BISPHOSPHONATES; GUIDELINES; HEALTH SERVICES RESEARCH

\section{Introduction}

ip fractures are associated with major distress, disability, - dependency, ${ }^{(1)}$ and significant costs to health and social care. ${ }^{(2)}$ Hip fracture patients are at increased risk of subsequent fracture, premature death, ${ }^{(1)}$ and a decline in quality of life that is comparable to severe neurological diseases. ${ }^{(3)}$ The projected cost of hip fracture for the year 2025 is approximately $\$ 18$ billion in the United States ${ }^{(4)}$ and $£ 1.5$ billion in the United Kingdom. ${ }^{(2)}$
Data from randomized controlled trials (RCTs) have showed that bisphosphonate treatment is highly effective for the secondary prevention of osteoporotic fracture, ${ }^{(5)}$ including among hip fracture patients. ${ }^{(6)}$ This is reflected in many clinical guidelines from around the world that recommend the use of bisphosphonate for patients having sustained a minimal trauma fracture. ${ }^{(7)}$ In January 2005, the National Institute for Health and Care Excellence (NICE) in England and Wales published new guidance recommending bisphosphonate use as a first-line

Received in original form January 15, 2016; revised form May 26, 2016; accepted May 29, 2016. Accepted manuscript online Month 00 , 2016.

Address correspondence to: Andrew Judge, PhD, NIHR Musculoskeletal Biomedical Research Unit, Nuffield Department of Orthopaedics, Rheumatology and Musculoskeletal Sciences, University of Oxford, Windmill Road, Headington, Oxford, OX3 7LD, UK. E-mail: andrew.judge@ndorms.ox.ac.uk

${ }^{*}$ AJ and MKJ are joint senior authors.

Additional Supporting Information may be found in the online version of this article.

Journal of Bone and Mineral Research, Vol. 31, No. 11, November 2016, pp 2008-2015

DOI: $10.1002 / j b m r .2882$

(C) 2016 American Society for Bone and Mineral Research 
therapy for the secondary prevention of osteoporotic fracture. ${ }^{(8)}$ Moreover, the first-time UK market authorization of generic bisphosphonate alendronic acid occurred in August 2005, after which the cost of therapy fell substantially.

We therefore aimed to evaluate these "real world" events within the English and Welsh National Health Service (NHS) setting in terms of subsequent change in antiosteoporosis medication prescribing and incidence of clinical outcomes such prescriptions were intended to prevent; ie, secondary osteoporotic fractures.

\section{Materials and Methods}

\section{Study population and source of data}

We used primary care data from the Clinical Practice Research Datalink (CPRD) for the period 1999 to 2013. CPRD covers approximately 11.3 million patients from 674 UK practices and has a current representative coverage of approximately $7 \%$ of the United Kingdom. ${ }^{(9)}$ Mortality data was linked to the Office for National Statistics (ONS) database. Primary hip fractures occurring between April 1, 1999 and September 30, 2012 were identified using standard UK clinical terminology "READ" codes (Supplementary Table 1) as defined a priori by two clinicians with experience in both clinical practice and epidemiological research into osteoporosis independently identifying available codes and reaching consensus. To focus on proximal femoral fractures, subtrochanteric/shaft fractures were not included. Patients below 60 years of age were excluded, as were patients with a history of a hip fracture diagnosis in the preceding 3 years or with less than 3 years of clinical data from registration. Patients registered in a general practitioner (GP) practice outside England or Wales were excluded as guidance pertained only to these countries.

\section{Intervention}

Publication of NICE TA $87^{(8)}$ in January 2005 and the first authorization of generic forms of alendronic acid in August 2005 were considered as a combined intervention because of their close temporal proximity. ${ }^{(10)}$ NICE TA 87 gave for the first time guidance on clinical thresholds for prescribing various antiosteoporosis medications among postmenopausal women having sustained a clinically apparent osteoporotic fracture (ie, in secondary prevention). It recommended the use of bisphosphonates for the treatment of women aged 75 years or older without the need for prior dual-energy X-ray absorptiometry (DXA) scanning. The authorization of generic alendronic acid led to a substantial reduction in price, to what is now approximately $£ 1$ per month. ${ }^{(11)}$

\section{Outcomes}

The proportion of patients initiating antiosteoporosis medications within 1 year of their hip fracture was calculated among treatment-naive patients (no such prescription within prior 6 months). Medications included were oral bisphosphonates, strontium ranelate, teriparatide, denosumab, and selective estrogen receptor modulators. Given concerns regarding low therapy adherence, we also modeled separately the proportion of patients who received at least one bisphosphonate prescription between 10 to 14 months after their hip fracture (excluding those patients not surviving to 14 months).

To examine the effect on re-fracture, we derived the proportion of patients (including prevalent users of antiosteoporosis medications) sustaining a subsequent major (hip, pelvis, proximal-humerus, rib, spine, or wrist/forearm) fracture within 3 years of their primary event. Hip fractures were only included if sustained between 6 and 36 months so as to avoid counting of re-coding events. Second hip fracture was also considered separately.

As a sensitivity analysis, we analyzed women separately because although treatment is typically offered to both genders following hip fracture, NICE TA 87 guidance pertained only to women. We repeated analyses excluding patients who either transferred out or were registered to a practice whose last data upload was within 1 year (for incident prescriptions time series) or 3 years (for re-fracture time series) of their primary hip fracture. We also repeated analyses for subsequent fracture outcomes stratified by therapy status at index hip-fracture.

\section{Statistical analysis}

We used an interrupted time series approach ${ }^{(12)}$ to estimate changes in outcomes immediately following the intervention period while controlling for baseline levels and trends. In order to take into account temporal changes in the age and sex structure of the population, we modeled aggregated data points in the form of age and sex standardized biannual proportions of each outcome of interest using segmented linear regression ${ }^{(10)}$ : $Y_{t}=\beta_{0}+\beta_{1}{ }^{*}$ time $_{t}+\beta_{2}{ }^{*}$ intervention $_{t}+\beta_{3}{ }^{*}$ post_int_time $t+e_{t}$. Here, $Y_{\mathrm{t}}$ is the proportion of index hip fracture patients with the outcome at time point (ie, 6-month period) $t$. $\beta_{0}$ estimates the baseline level of the outcome at the beginning of the time series. $\beta_{1}$ estimates the preintervention trend, $\beta_{2}$ the change in level immediately following the intervention, and $\beta_{3}$ the change in postintervention trend. The intervention period spanned a 1-year period, from the beginning of October 2004 to the end of September 2005. All analyses were therefore based on 11 preintervention data points (April 1999 to September 2004), and between 10 and 14 postintervention data points (October 2005 to September 2012) according to the follow-up time required for each outcome measure. Full models including all regression terms and final "conservative" models were derived, the latter by way of removing nonsignificant terms in a backward stepwise process ( $p$-entry $0.049 ; p$-exit 0.10 ). The presence of autocorrelation was tested using the Durban-Watson test. All Durban-Watson statistics were close to the value of 2 and above the higher bound; therefore, we did not reject the null hypothesis of no autocorrelation.

For ease of interpretation, we expressed regression coefficients for level and slope in the form of a single estimate of absolute change between estimated postintervention values and their counterfactual values ${ }^{(10,13)}$; ie, estimates for the same time point but based on preintervention level and trend only. We used the October 2007 to March 2008 time point because the end of March 2008 was the middle of the postintervention period (for the fracture time series). All analyses were performed using Stata v13.1 (Stata Corporation, Inc., College Station, TX, USA).

\section{Results}

We identified 10,873 eligible patients as having sustained an incident hip fracture within the period April 1999 to September 2012. There was an increase in the proportion of men $(21.8 \%$ versus $25.0 \%)$, proportion aged 85 years and older ( $41.9 \%$ versus $44.3 \%)$, severe rated comorbidities ( $2.4 \%$ versus $7.1 \%)$, and prior bisphosphonate use (5.8\% versus $24.2 \%)$ (Supplementary Table 2). 
Table 1. Outcomes of Interest Following Primary Hip Fracture: Stratified by Year $(n=10,873)$

\begin{tabular}{|c|c|c|c|c|c|c|c|c|c|}
\hline \multirow[b]{2}{*}{ Year (April-March) } & \multirow{2}{*}{$\frac{\text { Index hip fracture }}{n}$} & \multicolumn{2}{|c|}{$\begin{array}{c}\text { Incident } \\
\text { antiosteoporosis } \\
\text { medication } \\
\text { prescription } \\
(1-\text { year })^{a}\end{array}$} & \multicolumn{2}{|c|}{$\begin{array}{c}\geq 1 \\
\text { Bisphosphonate } \\
\text { prescription } \\
(10-14 \\
\text { months })^{\mathrm{b}}\end{array}$} & \multicolumn{2}{|c|}{$\begin{array}{l}\text { Subsequent } \\
\text { major fracture } \\
\left(^{(3 \text { years })^{c}}\right.\end{array}$} & \multicolumn{2}{|c|}{$\begin{array}{l}\text { Subsequent } \\
\text { hip fracture } \\
\text { (3 years) }^{c}\end{array}$} \\
\hline & & $n$ & $\%$ & $\mathrm{n}$ & $\%$ & $n$ & $\%$ & $n$ & $\%$ \\
\hline 1999 & 861 & 47 & 5.7 & 30 & 5.0 & 50 & 5.8 & 27 & 3.1 \\
\hline 2000 & 855 & 42 & 5.2 & 30 & 4.9 & 56 & 6.6 & 31 & 3.6 \\
\hline 2001 & 830 & 65 & 8.4 & 49 & 8.1 & 54 & 6.5 & 34 & 4.1 \\
\hline 2002 & 861 & 84 & 10.5 & 78 & 13.4 & 59 & 6.9 & 34 & 4 \\
\hline 2003 & 878 & 112 & 14 & 87 & 14.4 & 56 & 6.4 & 34 & 3.9 \\
\hline 2004 & 817 & 126 & 17.1 & 93 & 16.6 & 61 & 7.5 & 33 & 4 \\
\hline 2005 & 841 & 225 & 29.9 & 165 & 29.7 & 48 & 5.7 & 25 & 3 \\
\hline 2006 & 825 & 267 & 37.1 & 184 & 33.8 & 48 & 5.8 & 26 & 3.2 \\
\hline 2007 & 816 & 256 & 36.5 & 159 & 30.6 & 39 & 4.8 & 23 & 2.8 \\
\hline 2008 & 795 & 290 & 42.6 & 167 & 31.9 & 47 & 5.9 & 25 & 3.1 \\
\hline 2009 & 756 & 278 & 43.6 & 166 & 34.3 & 36 & 4.8 & 17 & 2.3 \\
\hline 2010 & 719 & 283 & 46.2 & 172 & 34.2 & $17^{d}$ & $4.6^{d}$ & $7^{d}$ & $1.9^{d}$ \\
\hline 2011 & 667 & 293 & 50.9 & 187 & 39.4 & & & & \\
\hline $2012^{d}$ & $352^{d}$ & $148^{d}$ & $48.8^{d}$ & & & & & & \\
\hline Overall & 10,873 & 2,516 & 25.9 & 1,599 & 21.8 & 571 & 6.0 & 316 & 3.3 \\
\hline
\end{tabular}

${ }^{a}$ Only among treatment-naive primary hip fracture patients (defined as no antiosteoporosis prescription in 6 months prior to index fracture).

${ }^{b}$ Only among bisphosphonate treatment naïve primary hip fracture patients surviving to 14 months (defined as no bisphosphonate prescription in 6 months prior to index fracture).

'Second hip fractures only counted between 6 and 36 months.

dBased on months April through September.

Table 1 reports study outcomes by financial year. Comparing years 1999-2000 to 2011-2012, the initiation of antiosteoporosis medication within 12 months increased markedly from $5.4 \%$ to $50.3 \%$, although there were significant differences by gender and age band (Fig. $1 A, B$ ). The overall increase was mainly driven by alendronic acid (Fig. 1C, D). Between 1999-2000 and 2009-2010, the proportion of patients sustaining a subsequent major or second hip fracture (as defined in Outcomes section) declined from $6.2 \%$ to $4.7 \%$, and $3.4 \%$ to $2.1 \%$, respectively.

Results from the interrupted time series analyses are reported as derived from parsimonious regression models. Regression coefficients from "full" models are provided in Supplementary Table 4.

\section{Prescription data}

We found a preintervention increase in antiosteoporosis prescriptions of $1.05 \%$ per 6 months of the study period, then a marked step change of $14.9 \%(95 \% \mathrm{Cl}, 10.9$ to $18.9 ; p \leq 0.001)$ taking place between preintervention and postintervention periods (Fig. 2A, Supplementary Table 3). Also found was a postintervention trend increase of $0.46 \%$ per 6 months $(95 \% \mathrm{Cl}$, -0.05 to $0.96 ; p=0.075)$. This equated to an overall estimated $17.2 \%(95 \% \mathrm{Cl}, 11.0$ to 23.3$)$ absolute increase in incident antiosteoporosis use within 1 year of hip fracture for the time point 3 years following the start of the intervention period (Table 2), representing an approximate relative increase of $79 \%$. Similar although slightly lower increases in prescribing were seen when restricted to $\geq 1$ bisphosphonate prescriptions between 10 and 14 months (Fig. 2B, Table 2, Supplementary Table 3).

\section{Subsequent major re-fracture and hip re-fracture}

The preintervention level of subsequent major re-fracture was stable at $6.3 \%(95 \% \mathrm{Cl}, 5.9$ to 6.8$)$. Following the intervention there was a significant $(p=0.001)$ downward trend by $-0.19 \%$ per 6 months $(95 \% \mathrm{Cl},-0.28$ to $-0.09 ; p=0.001)$ (Fig. $3 A)$. This equated to an absolute reduction of $-1.0 \%(95 \% \mathrm{Cl},-1.4$ to -0.5$)$ at 3 years after the start of the intervention period (Table 2), and an approximate relative $14 \%$ decrease. A similar reduction was demonstrated for subsequent hip fracture (Fig. $3 B$ ): an initial stable rate of $3.8 \%\left(95 \% \mathrm{Cl}_{1} 3.4\right.$ to 4.2$)$ followed by a postintervention downward trend of $-0.17 \%(95 \% \mathrm{Cl},-0.26$ to $-0.09 ; p=0.001)$ per 6 months. This equated to an absolute reduction of $-0.9 \% 3$ years following the start of the intervention period, and an approximate relative reduction of $22 \%$.

The same time series terms were included in final models in sensitivity analyses including only women (results not shown) and excluding patients with incomplete follow-up (Supplementary Table 5), with the exception that no trend change was detected in incident prescriptions and a significant step-change decrease in subsequent major fractures was found when patients with incomplete follow-up were excluded. We report analyses stratified by therapy status at time of index hip fracture in Supplementary Table 6 . For patients not on therapy at baseline, these were the same as for the main analysis. Among prevalent users, however, there was no significant impact of intervention identified, although this is likely to have been a consequence of the much smaller sample size in this subgroup, especially in the preintervention period.

\section{Discussion}

Our study identifies that following the introduction of NICE TA 87 and first UK market authorization of generic alendronic acid, prescriptions of antiosteoporosis medications increased markedly among hip fracture patients. This was contemporary 
(a): anti-OP initiation within 1-year from index hip fracture: stratified by gender

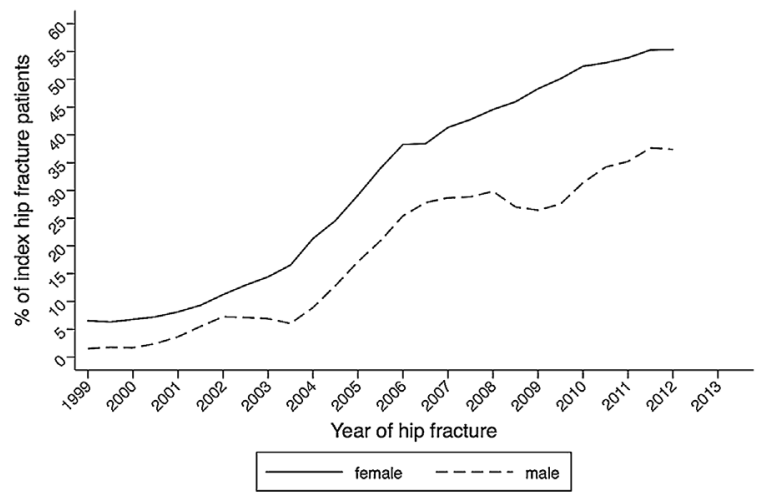

(c) : anti-OP initiation within 1-year: stratified by anti-OP medication type

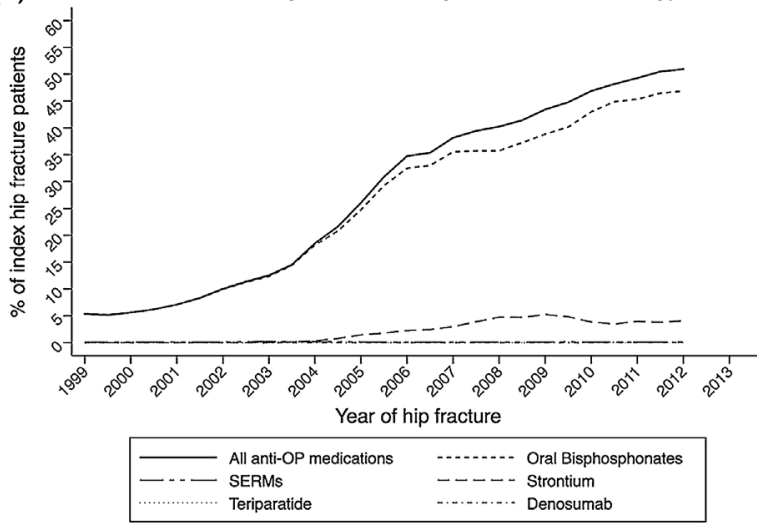

(b): anti-OP initiation within 1-year from index hip fracture: stratified by age

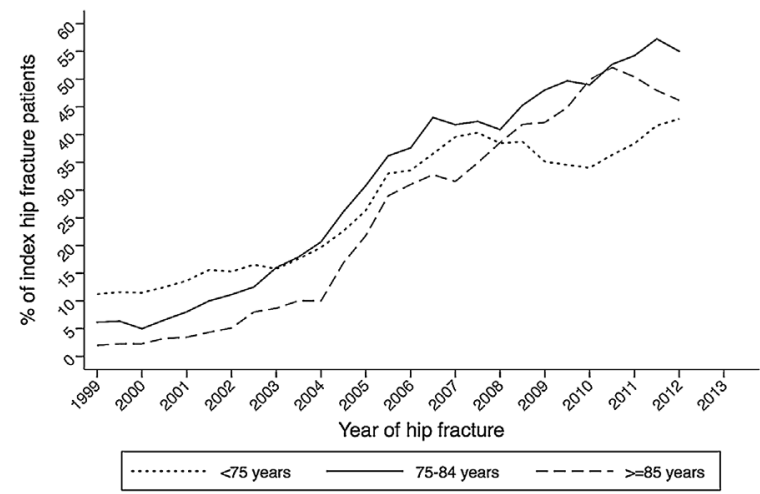

(d): anti-OP initiation within 1-year from index hip fracture: stratified by bisphosphonate type

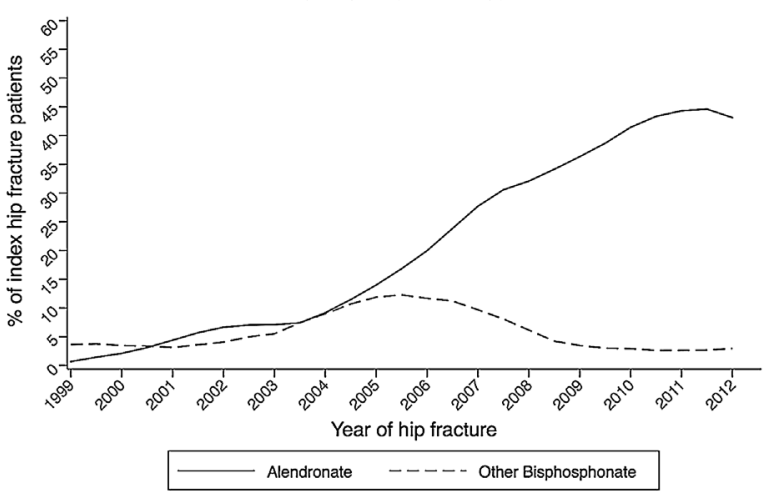

Fig. 1. Descriptive trends among primary hip fracture patients in: $(A)$ antiosteoporosis medication use within 12 months stratified by gender; $(B)$ antiosteoporosis medication use within 12 months stratified by age; $(C)$ antiosteoporosis medication use within 12 months stratified by type; and $(D)$ bisphosphonate use within 12 months stratified by type.

with a significant decline in the incidence of subsequent fractures and estimated hospital costs.

Considering the official and widespread use of NICE guidance within the NHS, the finding of significantly increased prescribing following NICE TA 87 is somewhat an expected finding and is consistent with previous reports of temporal associations between NICE publications and the health or prescription outcomes they were intended to impact. ${ }^{(14,15)}$ Although we did not disentangle the effect of the NICE TA 87 publication from availability of generic alendronic acid, the increase in prescriptions across genders and age groups (Fig. 1A, $B$ ) and divergence of alendronic acid versus other types of bisphosphonates (Fig. 1D) suggests availability of generic therapy was influential.

Although a decline in subsequent fracture incidence was concomitant with increased bisphosphonate prescribing, we have not here been able to establish whether increased prescribing caused the reduction in subsequent fractures. Therapy recommendations contained within NICE TA 87 were based on data from 39 published RCTs, ${ }^{(8)}$ with reference made to the Fracture Intervention Trial where the relative risk (RR) between alendronate versus placebo arms for subsequent "any clinical" or hip fracture was $0.74(95 \% \mathrm{Cl}, 0.59$ to 0.92$)$ and 0.49 (95\% Cl, 0.23 to 0.99 ), respectively, among women with an existing vertebral fracture. ${ }^{(16)}$ Our use of an interrupted time series approach that controls for baseline level and trend is considered a strong quasi-experimental modeling strategy, allowing for "real world" estimation of longitudinal effects when an RCT is unfeasible. ${ }^{(10,12)}$ The reverse of this ecological correlation has also been previously demonstrated, ie, recent increases in hip fracture incidence alongside a reduction in bisphosphonate use, ${ }^{(17)}$ itself likely due to reports of atypical femoral fractures associated with long-term bisphosphonate use. The large number of primary hip fractures and the generalizability of the CPRD cohort to the general UK population $^{(9)}$ are further strengths of the analysis.

One main limitation is that changes in outcomes may have been confounded by events other than the defined intervention. ${ }^{(1,18)}$ In this context, studies from other countries have identified downward trends in fracture rates to an extent that is beyond that attributable to increased bisphosphonate use alone, ${ }^{(19,20)}$ thereby prompting explanations of other contributory factors such as changes in BMI, smoking, and vitamin D status or improvement in falls prevention services. On the other hand, a significant decline in hip fracture incidence in Canada has previously been reported for the time period 1985 to $2005,{ }^{(21)}$ predating the era of large-scale antiosteoporosis medication use and therefore dependent on alternative explanations than improvement in treatment rates. One such candidate there suggested was a birth-cohort effect, which may have been a factor in the observed decline in our study were such a phenomenon to be concomitant with our defined intervention period. One issue is the speed of change in 
(a): Index hip fracture patients initiating anti-OP therapy (0-12 months)

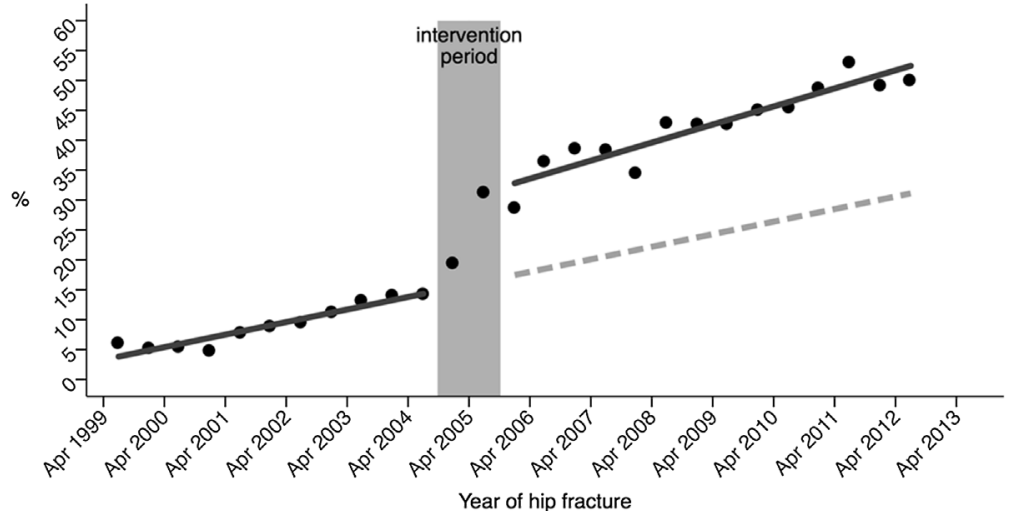

(b): Index hip fracture survivors with $\geq 1$ bisphosphonate prescription (12-14 months)

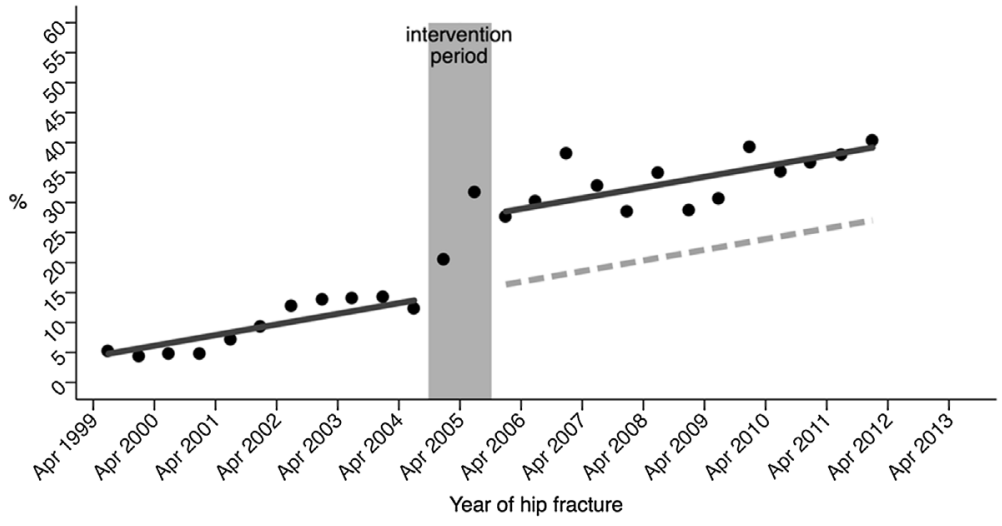

- observed bi-annual proportions (standardised)

estimated pre- and post-intervention trends based on observed values

$== \pm$ estimated post-intervention trend based on pre-intervention trend only

Fig. 2. (A) Results from segmented linear regression of incident antiosteoporosis medication in the first year after index hip fracture and $(B)$ results from segmented linear regression of bisphosphonate medication use between 10 and 14 months after index hip fracture, among treatment-naive patients at baseline who survived to 14 months.

Table 2. Estimated Absolute Change in Outcomes Under Study, Associated With the Publication of NICE TA 87 and First UK Market Authorization of Generic Alendronic Acid

\begin{tabular}{|c|c|c|c|c|c|}
\hline \multirow[b]{2}{*}{ Outcomes } & \multicolumn{2}{|c|}{ October 2007 to March 2008 estimate $^{a}$} & \multicolumn{3}{|c|}{ Absolute change $^{a}$} \\
\hline & $\begin{array}{l}\text { Without intervention } \\
\text { (\%) }\end{array}$ & $\begin{array}{c}\text { With intervention } \\
(\%)\end{array}$ & $\begin{array}{l}\text { Estimate } \\
(\%)^{\mathrm{e}}\end{array}$ & $\begin{array}{l}\text { Lower } \\
95 \% \mathrm{Cl}\end{array}$ & $\begin{array}{l}\text { Upper } \\
95 \% \mathrm{CI}\end{array}$ \\
\hline $\begin{array}{l}\text { Subsequent antiosteoporosis medication } \\
\qquad(0-12 \text { months })^{\mathrm{b}}\end{array}$ & 21.7 & 38.9 & 17.2 & 11.0 & 23.3 \\
\hline $\begin{array}{l}\text { Subsequent bisphosphonate medication } \\
\qquad(10-14 \text { months })^{b, c}\end{array}$ & 19.9 & 32.1 & 12.2 & 6.6 & 17.7 \\
\hline $\begin{array}{l}\text { Subsequent major fractures } \\
\qquad(0-36 \text { months })^{d}\end{array}$ & 6.3 & 5.4 & -1.0 & -1.4 & -0.5 \\
\hline Subsequent hip fractures (6-36 months) ${ }^{d}$ & 3.8 & 3.0 & -0.9 & -1.2 & -0.5 \\
\hline
\end{tabular}

Values calculated by comparing estimated values in the period October 2007 to March 2008 ( 3 years after the beginning of the intervention phase) to counterfactual values for the same period (ie, those expected for October 2007 to March 2008 based only on the preintervention level and trend).

${ }^{a}$ Estimated from final/parsimonious models specified using backward-stepwise selection ( $p$-entry 0.049 and $p$-exit 0.10 ). Percentages rounded to one decimal place.

${ }^{\mathrm{b}}$ Among treatment-naive index hip fracture patients.

${ }^{c}$ Among survivors at 14 months.

${ }^{\mathrm{d} S u b s e q u e n t}$ hip fractures only counted between 6 and 36 months. 
(a): Index hip fracture patients with subsequent major fracture (3-year)

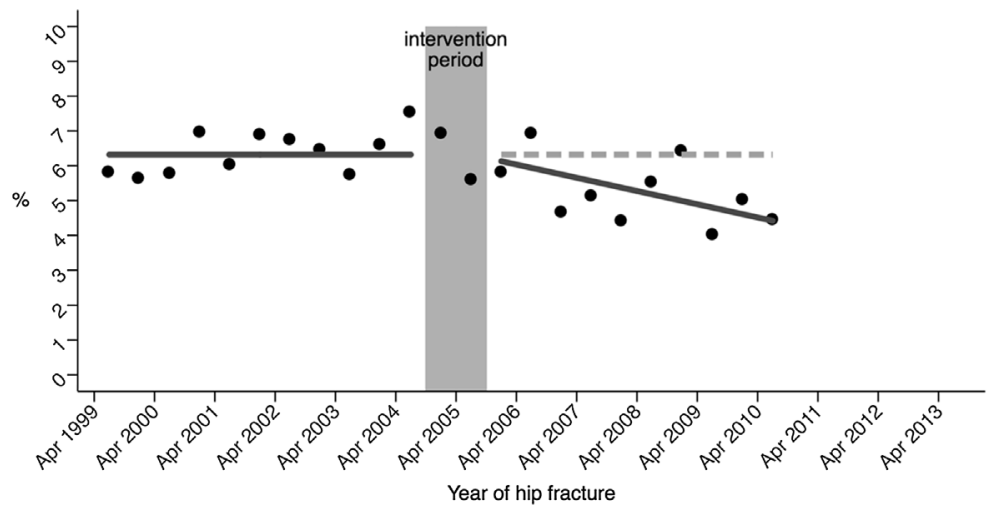

(b): Index hip fracture patients with subsequent hip fracture (3-year)

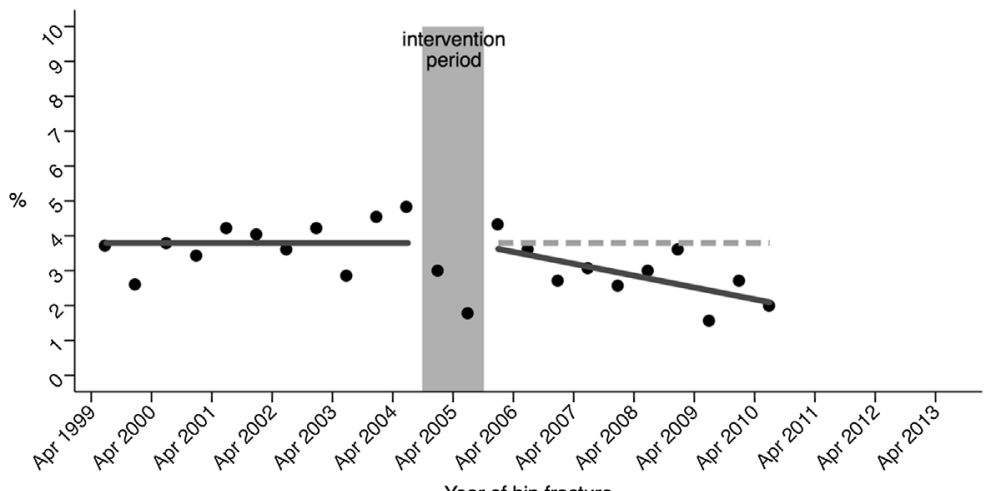

Year of hip fracture

- $\quad$ observed bi-annual proportions (standardised)

estimated pre- and post-intervention trend based on observed values

$==E$ estimated post-intervention trend based on pre-intervention trend only

Fig. 3. (A) Results from segmented linear regression of subsequent major fracture within 3 years after index hip fracture and $(B)$ results from segmented linear regression of subsequent hip fracture within 3 years (6-36 months) after index hip fracture.

prescription and re-fracture rates, which suggest that slower changes in demographics such as obesity are less likely to be the main cause of the findings of this study. Furthermore, lack of association between bisphosphonate use and hip fracture incidence across Canadian provinces has also recently been reported, ${ }^{(22)}$ although those data did not distinguish between primary and secondary prevention of fractures or address the issue of confounding by indication.

Although the discrete intervention evaluated here was the publication of NICE guidance and availability of generic alendronate, other changes in the health care system such as in the introduction of Fracture Liaison Service (FLS) models of care $^{(23,24)}$ may also have contributed to the findings. Given that the FLS operates to improve case-finding, assessment, therapy initiation, and monitoring after a fragility fracture and that such services have become increasingly common in the NHS, it is plausible these models of care contributed to the steeper and sustained postintervention increase in prescribing of the therapies which were recommended and became substantially cheaper during the intervention period. An economic evaluation of an FLS in the UK health setting has shown FLS to be a costeffective model for improving secondary fracture prevention within the United Kingdom, ${ }^{(25)}$ although the fracture reduction was based on prescribing rates only. Other studies have reported no observed difference in fracture rates by FLS status, $^{(26)}$ one possible explanation being poor medication adherence. Further work is required given the wider availability of parenteral therapies with significantly greater adherence rates. ${ }^{(27)}$

It is worth noting that in a post hoc analysis we found our decline in 3-year re-fracture rate to be occurring in the context of a stable 3-year mortality rate, although 1-year mortality did improve over the course of the study period ( $25.3 \%$ versus $19.8 \%$ for years 1999-2000 to 2012-13). However, given that fracture risk after hip fracture is high, improved survival at 1-year would likely increase rather than reduce the re-fracture rates as patients live longer at an elevated risk of re-fracture. ${ }^{(28)}$ Acknowledging that persistence with bisphosphonates is poor, ${ }^{(29)}$ it is reassuring that the intervention was associated here with increased prescribing between 12 and 14 months.

Reporting absolute difference between estimated postintervention values compared to estimated contralateral values does involve extrapolation and therefore uncertainty, although 95\% confidence intervals incorporating the standard error of regression coefficients are included. ${ }^{(13)}$ We also had more than the minimum number of eight time points before and after the 
intervention to have sufficient power to estimate the regression coefficients. ${ }^{\left({ }^{30}\right)}$ Main findings were derived from parsimonious regression models, but estimated changes in fracture outcomes were larger when "full" models were used (Supplementary Fig. 1, Supplementary Table 4). Using routinely collected data with no individual validation of fracture events is another limitation; however, validation of hip and vertebral fracture coding has been carried out previously and has been shown to be accurate. ${ }^{(31)}$ Our definition of second hip fracture is based on previous work $^{(32)}$ and incorporates a 6-month washout from the index fracture to minimize the inclusion of recoding events, although such occurrences cannot be ruled out.

Although we set out to investigate the potential impact on prescribing and the clinical outcomes such prescribing was intended to improve, we did not model the unintended consequences of bisphosphonate use. Widespread concern exists regarding adverse events (eg, gastrointestinal problems) and safety issues associated with bisphosphonate use, most notably osteonecrosis of the jaw and atypical fractures. ${ }^{(33)}$ However, given that the absolute risk of these safety issues is low at approximately one in $\mathbf{3 0 0}$ patients sustaining an atypical fracture among those treated for 3 years ${ }^{(34)}$ and the high rate of discontinuation among bisphosphonate users, ${ }^{(29)}$ we would be underpowered to investigate such events here. Furthermore, although we acknowledge that adverse events from the use of some antiosteoporosis medications are important to include, there is no READ code for atypical fractures and so this could not be included in the model and remains an area for future research.

Our time series of prescriptions data is consistent with secular trends of similar outcomes reported in the literature. ${ }^{(35)}$ Despite the observed increase in prescribing, our findings support previous reports of undertreatment of high-risk patients, contrary to guideline recommendations. ${ }^{(36,37)}$ For example, a national audit within the United Kingdom indicated in 2010 that $40 \%$ of hip fracture patients did not receive appropriate bone health treatment. ${ }^{(38)}$ One caveat here is that we were not able to consider zoledronic acid use because it is rarely administered in a primary care setting.

Exact comparison of re-fracture rates to other studies is difficult because of the difference in definitions, outcome measures, and time periods used. ${ }^{(26,39,40)}$ Particularly, our UK cohort of hip fractures was smaller and our secular trend in re-fracture different from that reported elsewhere, ${ }^{(41)}$ although in order to be highly specific that we included true incident hip fractures, our inclusion criteria required 3 years' registration in a CPRD-participating GP practice and only considered proximal femoral fractures. However, we may have created an artificially stable study sample with respect to GP practice in only including those with 3 years of GP registration prior to their index hip fracture.

In summary, although this is an observational study and we have not proven a cause-effect relationship, we have shown that among hip fracture patients, publication of NICE guidance and availability of generic alendronic acid was temporally associated with an increase in antiosteoporosis medication prescribing and a clinically important reduction in the incidence of subsequent fracture.

\section{Disclosures}

JL, DPA, NKA, CC, MKJ, and AJ received grants from NIHR Health Services and Delivery Research (HS\&DR) during the conduct of the study. Outside the submitted work, MKJ reports personal fees from Lilly UK, Amgen, Sevier, Merck, Medtronic, Internis, and Consilient Health, and serves on the Scientific Committee of the National Osteoporosis Society and International Osteoporosis Foundation; DPA received grants from Bioiberica S.A. and Amgen Spain S.A.; CC received personal fees from Servier, Amgen, Eli Lilly, Merck, Medtronic, and Novartis. NKA reports personal fees from Merck, Smith and Nephew, Q-Med, Nicox, Flexion, Bioiberica, and Servier, and grants and personal fees from Roche. AJ has received consultancy, lecture fees, and honoraria from Servier, UK Renal Registry, Oxford Craniofacial Unit, IDIAP Jordi Gol, and Freshfields Bruckhaus Deringer, and has held advisory board positions (which involved receipt of fees) from Anthera Pharmaceuticals, Inc., and received research sponsorship from Roche. $\mathrm{SH}$ and $\mathrm{AD}$ have no competing financial interests relevant to the submitted work.

\section{Acknowledgments}

This project was funded by the NIHR HS\&DR program (project number 11/1023/01). Partial support was also received from the Oxford NIHR Musculoskeletal Biomedical Research Unit, Nuffield Orthopaedic Centre, University of Oxford. The funding source had no role in the design and conduct of the study, in the collection, analysis, and interpretation of the data, or in the preparation, review, or approval of the manuscript. The views and opinions expressed therein are those of the authors and do not necessarily reflect those of the HS\&DR program, NIHR, NHS, or the Department of Health. This study is based in part on data from the Clinical Practice Research Datalink obtained under license from the UK Medicines and Healthcare products Regulatory Agency.

The ReFRESH study group consists of Dr Andrew David Judge, Dr Muhammad Kassim Javaid, Professor Nigel Arden, Professor Cyrus Cooper, Professor Andrew Farmer, Dr Daniel Prieto-Alhambra, Dr Jose Leal, Professor Michael Goldacre, Professor Alastair Gray, Dr Janet Lippett, Dr Rachael GoobermanHill, and Laura Graham.

Authors' roles: $\mathrm{AJ}, \mathrm{MKJ}$, and $\mathrm{SH}$ take responsibility for design of the study. $\mathrm{SH}$ and $\mathrm{AJ}$ take responsibility for the integrity of the prescriptions and incidence data and the accuracy of the statistical analysis. AD takes responsibility for data management. All authors provided inputs and comments to the article and approved the final version. AJ and MKJ supervised the study and are joint senior authors.

\section{References}

1. British Orthopaedic Association (BOA). The care of patients with fragility fractures [Internet]. London (UK): BOA; 2007 [cited 2016 Jun 23]. Available from: http://www.bgs.org.uk/pdf_cms/pubs/Blue Book on fragility fracture care.pdf.

2. Leal J, Gray AM, Prieto-Alhambra D, et al. Impact of hip fracture on hospital care costs: a population-based study. Osteoporos Int. 2016;27(2):549-58.

3. Griffin XL, Parsons N, Achten J, Fernandez M, Costa ML. Recovery of health-related quality of life in a United Kingdom hip fracture population. The Warwick Hip Trauma Evaluation-a prospective cohort study. Bone Joint J. 2015;97-B(3):372-82.

4. Burge R, Dawson-Hughes $B$, Solomon DH, Wong JB, King A, Tosteson A. Incidence and economic burden of osteoporosis-related fractures in the United States, 2005-2025. J Bone Miner Res. 2007;22(3): 465-75.

5. Wells GA, Cranney A, Peterson J, et al. Alendronate for the primary and secondary prevention of osteoporotic fractures in postmenopausal women. Cochrane Database Syst Rev. 2008;(1):CD001155. 
6. Lyles KW, Colon-Emeric CS, Magaziner JS, et al. Zoledronic acid and clinical fractures and mortality after hip fracture. $\mathrm{N}$ Engl J Med. 2007;357(18):1799-809.

7. Ganda K, Puech M, Chen JS, et al. Models of care for the secondary prevention of osteoporotic fractures: a systematic review and metaanalysis. Osteoporos Int. 2013;24(2):393-406.

8. National Institute for Health and Care Excellence (NICE). Bisphosphonates (alendronate, etidronate, risedronate), selective oestrogen receptor modulators (raloxifene) and parathyroid hormone (teriparatide) for the secondary prevention of osteoporotic fragility fractures in postmenopausal women. Technological Appraisal 87. London, UK: National Institute for Health and Care Excellence (NICE); 2005.

9. Herrett E, Gallagher AM, Bhaskaran K, et al. Data Resource Profile: Clinical Practice Research Datalink (CPRD). Int J Epidemiol. 2015 Jun;44(3):827-36.

10. Wagner AK, Soumerai SB, Zhang F, Ross-Degnan D. Segmented regression analysis of interrupted time series studies in medication use research. J Clin Pharm Ther. 2002;27(4):299-309.

11. British National Formulary. Alendronic Acid (Non-proprietary) [Internet]: British National Formulary; 2015 [cited 2016 Jun 23]. Available from: https://www.evidence.nhs.uk/formulary/bnf/current/ 6-endocrine-system/66-drugs-affecting-bone-metabolism/662bisphosphonates-and-other-drugs-affecting-bone-metabolism/ bisphosphonates/alendronic-acid/alendronic-acid.

12. Kontopantelis E, Doran T, Springate DA, Buchan I, Reeves D. Regression based quasi-experimental approach when randomisation is not an option: interrupted time series analysis. BMJ. 2015; 350:h2750.

13. Zhang F, Wagner AK, Soumerai SB, Ross-Degnan D. Methods for estimating confidence intervals in interrupted time series analyses of health interventions. J Clin Epidemiol. 2009;62(2):143-8.

14. Audit NP. Impact of NICE guidance on rates of haemorrhage after tonsillectomy: an evaluation of guidance issued during an ongoing national tonsillectomy audit. Qual Saf Health Care. 2008;17(4):264-8.

15. Dayer MJ, Jones S, Prendergast B, Baddour LM, Lockhart PB, Thornhill $\mathrm{MH}$. Incidence of infective endocarditis in England, 2000-13: a secular trend, interrupted time-series analysis. Lancet. 2015;385(9974): 1219-28

16. Black DM, Thompson DE, Bauer DC, et al. Fracture risk reduction with alendronate in women with osteoporosis: the Fracture Intervention Trial. FIT Research Group. J Clin Endocrinol Metab. 2000;85(11): 4118-24

17. Fisher A, Martin J, Srikusalanukul W, Davis M. Bisphosphonate use and hip fracture epidemiology: ecologic proof from the contrary. Clin Interv Aging. 2010;5:355-62.

18. National Institute for Clinical Excellence (NICE). Falls: assessment and prevention of falls in older people. Clinical Guideline 21. London, UK: NICE; 2004.

19. Abrahamsen B, Vestergaard P. Declining incidence of hip fractures and the extent of use of anti-osteoporotic therapy in Denmark 19972006. Osteoporos Int. 2010;21:373-80.

20. Korhonen N, Niemi S, Parkkari J, Sievanen H, Palvanen M, Kannus P. Continuous decline in incidence of hip fracture: nationwide statistics from Finland between 1970 and 2010. Osteoporos Int. 2013;24(5): 1599-603.

21. Leslie WD, O'Donnell $S$, Jean $S$, et al. Trends in hip fracture rates in Canada. JAMA. 2009:302(8):883-9.

22. Crilly RG, Kloseck M, Chesworth B, Mequanint S, Sadowski E, Gilliland J. Comparison of hip fracture and osteoporosis medication prescription rates across Canadian provinces. Osteoporos Int. 2014; 25(1):205-10.

23. Drew $S$, Sheard $S$, Chana J, et al. Describing variation in the delivery of secondary fracture prevention after hip fracture: an overview of 11 hospitals within one regional area in England. Osteoporos Int. 2014:25(10):2427-33.
24. Drew S, Judge A, Cooper C, Javaid MK, Farmer A, Gooberman-Hill R Secondary prevention of fractures after hip fracture: a qualitative study of effective service delivery. Osteoporos Int. 2016 May;27(5):1719-27.

25. McLellan AR, Wolowacz SE, Zimovetz EA, et al. Fracture liaison services for the evaluation and management of patients with osteoporotic fracture: a cost-effectiveness evaluation based on data collected over 8 years of service provision. Osteoporos Int. 2011;22(7):2083-98.

26. Hawley S, Javaid MK, Prieto-Alhambra D, et al. Clinical effectiveness of orthogeriatric and fracture liaison service models of care for hip fracture patients: population-based longitudinal study. Age Ageing. 2016;45(2):236-42.

27. Hadji P, Papaioannou N, Gielen E, et al. Persistence, adherence, and medication-taking behavior in women with postmenopausal osteoporosis receiving denosumab in routine practice in Germany, Austria, Greece, and Belgium: 12-month results from a European non-interventional study. Osteoporos Int. 2015;26(10):2479-89.

28. Curtis JR, Arora T, Matthews RS, et al. Is withholding osteoporosis medication after fracture sometimes rational? A comparison of the risk for second fracture versus death. J Am Med Dir Assoc. 2010;11(8):584-91.

29. Li L, Roddam A, Gitlin M, et al. Persistence with osteoporosis medications among postmenopausal women in the UK General Practice Research Database. Menopause. 2012;19(1):33-40.

30. Penfold RB, Zhang F. Use of interrupted time series analysis in evaluating health care quality improvements. Acad Pediatr. 2013;13(6):S38-44.

31. Van Staa TP, Abenhaim L, Cooper C, Zhang B, Leufkens HGM. The use of a large pharmacoepidemiological database to study exposure to oral corticosteroids and risk of fractures: Validation of study population and results. Pharmacoepidemiol Drug Saf. 2000;9(5):359-66.

32. Hawley S, Javaid MK, Rubin $\mathrm{KH}$, et al. Incidence and predictors of multiple fractures despite high adherence to oral bisphosphonates: a binational population-based cohort study. J Bone Miner Res. 2016 Jan;31(1):234-44.

33. Järvinen TL, Michaëlsson K, Jokihaara J, et al. Overdiagnosis of bone fragility in the quest to prevent hip fracture. BMJ. 2015 May 26;350: h2088.

34. Järvinen TL, Michaëlsson K, Aspenberg P, Sievänen H. Osteoporosis: the emperor has no clothes. J Intern Med. 2015;277(6):662-73.

35. Klop C, Gibson-Smith D, Elders PJ, et al. Anti-osteoporosis drug prescribing after hip fracture in the UK: 2000-2010. Osteoporos Int. 2015;26(7):1919-28.

36. Elvey MH, Pugh H, Schaller G, Dhotar G, Patel B, Oddy MJ. Failure in the application of fragility fracture prevention guidelines. Ann R Coll Surg Engl. 2014;96(5):381-5.

37. Feldstein A, Elmer PJ, Orwoll E, Herson M, Hillier T. Bone mineral density measurement and treatment for osteoporosis in older individuals with fractures-a gap in evidence-based practice guideline implementation. Arch Intern Med. 2003;163(18):2165-72.

38. Tremi J HJ, Lowe D, Vasilakis N. Falling standards, broken promises: report of the national audit of falls and bone health in older people [Internet]. London, UK: Royal College of Physicians; 2011 [cited 2016 Jun 23]. Available from: https://www.nos.org.uk/document.doc? id $=1516$.

39. Omsland TK, Holvik K, Meyer HE, et al. Hip fractures in Norway 19992008: time trends in total incidence and second hip fracture rates: a NOREPOS study. Eur J Epidemiol. 2012;27(10):807-14.

40. Melton LJ 3rd, Kearns AE, Atkinson EJ, et al. Secular trends in hip fracture incidence and recurrence. Osteoporos Int. 2009;20(5): 687-94.

41. Gibson-Smith D, Klop C, Elders PJM, et al. The risk of major and any (non-hip) fragility fracture after hip fracture in the United Kingdom: 2000-2010. Osteoporosis Int. 2014;25(11):2555-63. 